\title{
Finite-Time Synchronization of Uncertain Complex Dynamic Networks with Nonlinear Coupling
}

\author{
Yiping Luo $\mathbb{D}$ and Yuejie Yao \\ Hunan Institute of Engineering, Xiangtan, Hunan 411101, China \\ Correspondence should be addressed to Yiping Luo; lyp@hnie.edu.cn
}

Received 16 February 2019; Revised 3 June 2019; Accepted 20 August 2019; Published 20 December 2019

Academic Editor: Christos Volos

Copyright () 2019 Yiping Luo and Yuejie Yao. This is an open access article distributed under the Creative Commons Attribution License, which permits unrestricted use, distribution, and reproduction in any medium, provided the original work is properly cited.

\begin{abstract}
The finite-time synchronization control is studied in this paper for a class of nonlinear uncertain complex dynamic networks. The uncertainties in the network are unknown but bounded and satisfy some matching conditions. The coupling relationship between network nodes is described by a nonlinear function satisfying the Lipchitz condition. By introducing a simple Lyapunov function, two main results regarding finite-time synchronization of a class of complex dynamic networks with parameter uncertainties are derived. By employing some analysis techniques like matrix inequalities, suitable controllers can be designed based on the obtained synchronization criteria. Moreover, with the obtained control input, the time instant required for the system to achieve finite-time synchronization can be estimated if a set of LMIs are feasible or an assumption on the eigenvalues of some matrices can be satisfied. Finally, the effectiveness of the proposed results is verified by numerical simulation.
\end{abstract}

\section{Introduction}

Synchronization is a very important nonlinear phenomenon in nature. It means that two or more time-varying quantities maintain a certain relative relationship during the change process. As we know, a large number of systems, such as neural networks [1], social systems, electrical power systems [2], and the Internet, can be described by complex networks in real life. Synchronization, as one of the most significant characteristics of a network, has been a hot topic in the field of complex networks [3-6]. Due to its difficulty, the study of synchronization of a complex network remains challenging [7]. Up to now, fruitful results have emerged on this issue, see, e.g., [8-11].

Establishing an appropriate network model in accordance with the physical system's network characteristics is the basis of the study of complex network. In some actual complex network models, the internal coupling between network nodes is assumed to be a linear relation [8]. The relationship among the factors within the nodes of a complex network system is likely to be nonlinear. For example, the inner relationship among users, as the nodes of a mobile user network, is nonlinear. Therefore, the research on the inner coupling and nonlinear relationship between the nodes of a complex network is of great importance. At present, some research results about this problem are available [9, 10]. However, many problems still require further study. Moreover, the synchronization state may be affected and even destroyed due to external disturbances, fluctuations in system parameters, and other uncertain factors [12-15]. In a word, the research on the synchronization control of uncertain complex networks not only supports the theoretical results but also expands the value of such method in practical applications. To our knowledge, relevant research results are abundant [16-20]. Wang et al. [16] studied the exponential synchronization problem of a class of Markov jumping complex dynamic networks with partially uncertain transition rate and stochastic disturbances using the containment control method. In [17], Rakkiyappan et al. realized the exponential pulse projection and lag synchronization between uncertain complex networks by controlling some nodes and reduced the time required to achieve synchronization. Zhao and Cai [18] researched the exponential synchronization of complex 
delayed dynamic networks with uncertain parameters by using the method of intermittent control. Wu and Liu [19] obtained some sufficient conditions that can be used to allow uncertain complex dynamic networks to achieve exponential synchronization through adaptive control. Lu et al. [20] proposed two types of sufficient conditions for the global asymptotical synchronization of delayed complex dynamic networks with switching topology based on the single Lyapunov and multiple Lyapunov function methods. Syed Ali et al. [21] studied a class of Markovian jump complex dynamic networks with parameter uncertainties. However, those works only investigated the conditions of synchronization of uncertain complex network systems in infinite time.

Exponential synchronization has a high convergence rate, but it reaches the required synchronization state in an infinite time. In many practical applications, systems are expected to realize synchronization in a finite time to ensure their superior performance. Hence, the study of the finite-time synchronization of complex networks has received extensive attention. In recent years, the finite-time synchronization of complex networks has been studied by various researchers and important results have been attained. For example, Zhao et al. [22] proposed the finitetime synchronization for multilink complex networks via discontinuous control. Zhang et al. [23, 24] obtained several sufficient conditions for the finite-time synchronization of complex networks with nonidentical nodes under impulsive disturbances and stochastic noise perturbations. In [25], the problem of finite-time synchronization of complex dynamic networks with time-varying and nonidentical nodes was researched. Sun et al. [26] analyzed the finite-time synchronization problem of two complex chaotic systems with unknown parameters, which mainly used a nonsingular terminal sliding control method. Sheng et al. [27] studied the finite-time outer-synchronization of complex networks with Markov jump topology by using the method that combines random jump control and deterministic switch control. However, the results of the comprehensive analysis of finite-time synchronization are generally obtained on the basis of deterministic models of complex network systems.

In summary, the research on the finite-time synchronization of uncertain complex networks is relevant for theoretical development and practical applications. However, few studies explore the finite-time synchronization of uncertain complex networks. Xiao et al. [28] studied the finite-time cluster synchronization of a complex dynamic network with time-varying delay by using the hybrid control strategy; literature [29] used the average dwell time method to obtain some sufficient conditions for realizing finite-time synchronization of sampled-data T-S fuzzy complex dynamic networks. But, both of them considered the case where the relationship between the nodes is linearly coupled, and they do not consider the uncertainties that may be contained in the system. Zhang and Han [30] researched the finite-time synchronization of uncertain complex networks with nonidentical nodes on the basis of special unilateral coupling control; however, the method requires the estimation of uncertain parameters and the assumption of a topology that satisfies conditions automatically. Mei et al. [31] discussed a class of finite-time synchronization for drive-response systems with structure identification and uncertain parameters. Xu et al. [32] studied the finite-time synchronization of Markovian jump complex networks with generally uncertain transition rates. Ma et al. [33] studied the robust and nonfragile finite-time $H_{\infty}$ synchronization control for complex networks with uncertain inner coupling. Zhao et al. [34] obtained new results on finite-time parameter identification and synchronization of uncertain complex dynamic networks with perturbations and impulsive effects on the basis of the finite time stability theorem, in which a system's uncertainty is caused by uncertain parameters. Moreover, in [31-34], the nonlinearity of the internal coupling of systems was not considered. Sun et al. [35] realized the finite-time synchronization of complex chaotic systems with three unknown parameter terms by means of sliding mode control. In [36], the finite-time synchronization between two complex networks without uncertainty was proposed. However, the individual couplings were considered as linear, and the state vector of the ith dynamic node was not included. Li et al. [37] investigated the finite-time synchronization for a class of complex networks with nonidentical nodes and uncertain disturbance, but the system studied was linearly coupled, and the disturbance terms were assumed to satisfy the upper bound condition, without considering the possible disturbance factors of the nodes themselves.

Motivated by the abovementioned analysis, we study the problem of finite-time synchronization control for nonlinear uncertain complex dynamic networks. In the real world, the coupling within many network nodes is nonlinear. For example, when a network node is a neuron, the state components within the neuron share a typical nonlinear relationship. Therefore, this study considers a complex network model, in which the coupling between the nodes is nonlinear and the nonlinear vector function satisfies the Lipschitz condition. The innovations of this work are described as follows:

(1) A finite-time synchronization problem for a complex network model with uncertain parameters and nonlinear coupling inside the nodes is studied. The parameter uncertainties satisfy the matching condition. This assumption is based on practical problems and is nontrivial.

(2) A finite-time synchronization control strategy is proposed for this type of model and sufficient conditions for the finite-time synchronization are obtained. First, under an LMI condition, the time required for the system to achieve finite-time synchronization can be obtained with a given input. Second, under another assumption on the eigenvalues of some matrices, an upper bound for the synchronization time is obtained under another input. 
(3) A simple and feasible controller is designed for the model studied. Under the input of the controller, the time required for the system to reach the finite-time synchronization state is only related to the system parameters.

The remainder of this paper is organized as follows: In Section 2, the model of an uncertain complex dynamic network with nonlinear coupling and some preliminaries are introduced. In Section 3, some sufficient conditions for the system to realize finite-time synchronization are obtained. In Section 4, several numerical examples are given to verify the effectiveness of the theoretical results.

\section{System Description and Preliminaries}

In this paper, we consider a time-varying uncertain complex dynamic network composed of $N$ nonlinear decentralized coupling nodes, where each node is an $n$-dimensional dynamic system. The equation of state for node $i$ is described as follows:

$$
\begin{aligned}
\dot{x}_{i}(t)= & (A+\Delta A(t)) x_{i}(t)+(B+\Delta B(t)) f\left(x_{i}(t)\right) \\
& +\sum_{j=1}^{N} c_{i j} g\left(x_{j}(t)\right)+u_{i}(t), \quad i=1,2, \ldots, N,
\end{aligned}
$$

where $x_{i}(t)=\left(x_{i 1}(t), x_{i 2}(t), \ldots, x_{i n}(t)\right)^{T} \in R^{n}$ and $u_{i}(t)$ are the state vector and the control input vector of the $i$ th node, respectively; $f(x) \in R^{n}$ is a continuously differentiable nonlinear vector function describing the dynamic characteristics of nodes; $g(x) \in R^{n}$ is a smooth nonlinear vector function representing the internal coupling between the state variables of each node; $C=\left(c_{i j}\right)_{N \times N}$ is the parameter matrix of external coupling structure, which represents the topology and coupling strength of the network. If a connection exists between node $i$ and node $j$, then $c_{i j} \neq 0$; otherwise, $c_{i j}=0$. The diagonal elements of the matrix $C$ are given by $c_{i i}=-\sum_{j=1, i \neq j}^{N} c_{i j}$. The matrices $A$ and $B$ are constant matrices, and $\Delta A(t)$ and $\Delta B(t)$ are the norm bounded uncertainty matrices of the system with the following form:

$$
[\Delta A(t), \Delta B(t)]=H F(t)\left[E_{1}, E_{2}\right],
$$

where $H, E_{1}$, and $E_{2}$ are real known constant matrices of appropriate dimensions and $F(t)$ is the unknown timevarying matrix function satisfying $F^{T}(t) F(t) \leq I$.

Remark 1. Many studies on nonlinear complex network models assume that the dynamic behavior function of nodes is a noncoupling nonlinear function. However, in some networks, the dynamic characteristics between their nodes are interconnected. A typical example is a neural network. The coefficient matrix of the dynamic behavior function is not an identity matrix. This type of model is studied in the literature $[17,38,39]$.
Remark 2. The complex dynamic network model in the current study involves a few cases in which the parameters are uncertain. These cases are not widely studied in the literature $[17,33,38-41]$. For example, the authors in $[17,33,40]$ considered that the internal coupling matrix of a complex network model is uncertain. Although the literature [35] considers the parameter uncertainty, the model is a kind of neural networks model. In [36], a case of incomplete transmission factors was presented. In fact, the coupling of the nodes of a complex network model has a nonlinear relationship, and the findings of the literature $[17,33,40]$ can be combined with the special case of the model in the present study.

Definition 1 (see [42]). The complex dynamic network (1) is said to be globally synchronized, if there is a control input $u_{i}(t), i=1,2, \ldots, N$, such that

$$
\left\|x_{i}(t)-s(t)\right\|=0, \quad t \longrightarrow \infty, i=1,2, \ldots, N,
$$

where $s(t) \in R^{n}$ is the solution of the isolated node system $\dot{s}(t)=(A+\Delta A(t)) s(t)+(B+\Delta B(t)) f(s(t))$.

Furthermore, if there exists a constant $t^{*}>0$, such that $\lim _{t \rightarrow t^{*}}\left\|e_{i}(t)\right\|=0, \quad\left\|e_{i}(t)\right\| \equiv 0$ for any $t \geq t^{*}$, where $e_{i}(t)=x_{i}(t)-s(t)$, we can say that system (1) is finite-time synchronized.

Definition 2 (see [43]). Consider $A=\left(a_{i j}\right) \in c^{m \times n}$ and $B=\left(b_{i j}\right) \in c^{p \times q}$. Then, $A \otimes B=C^{m p \times n q}$ is called the Kronecker product of matrix $A$ and $B$.

Next, we list a number of key properties which could be occurred in the proof:

(1) $(A \otimes B)^{T}=A^{T} \otimes B^{T}$

(2) Suppose that $A=\left(a_{i j}\right)_{m \times n}, B=\left(b_{i j}\right)_{s \times r}, C=\left(c_{i j}\right)_{n \times p}$, and $D=\left(d_{i j}\right)_{r \times t}$, then $(A \otimes B)(C \otimes D)=A C \otimes B D$

Let $e_{i}(t)=x_{i}(t)-s(t)$ be the synchronization error vectors $i=1,2, \ldots, N$. Then, the synchronization error system can be given by

$$
\begin{aligned}
\dot{e}_{i}(t)= & (A+\Delta A(t)) e_{i}(t)+(B+\Delta B(t))\left(f\left(x_{i}(t)\right)-f(s(t))\right) \\
& +\sum_{j=1}^{N} c_{i j} \phi\left(e_{j}\right)(t)+u_{i}(t) .
\end{aligned}
$$

Set $\phi\left(e_{i}\right)(t)=g\left(x_{i}(t)\right)-g(s(t))$. This study aims to design a suitable feedback controller $u_{i}(t)$ of the following form such that the system (1) achieves finite-time synchronization:

$$
u_{i}(t)=-w_{i} e_{i}(t)-k \operatorname{sign}\left(e_{i}(t)\right)\left|e_{i}(t)\right|^{\beta},
$$

where $w_{i}(i=1,2, \ldots, N)$ and $k$ are real constants and $0<\beta<1$, and 


$$
\begin{aligned}
\left|e_{i}(t)\right|^{\beta} & =\left(\left|e_{i 1}(t)\right|^{\beta},\left|e_{i 2}(t)\right|^{\beta}, \ldots,\left|e_{i n}(t)\right|^{\beta}\right)^{T}, \\
\operatorname{sign}\left(e_{i}(t)\right) & =\left\{\begin{array}{cc}
1, & e_{i}(t)>0, \\
0, & e_{i}(t)=0, \\
-1, & e_{i}(t)<0 .
\end{array}\right.
\end{aligned}
$$

Assumption 1. For any $x(t), s(t) \in R^{n}$, there exists a positive constant $L$ such that

$$
\|f(x(t))-f(s(t))\| \leq L\|x(t)-s(t)\| .
$$

Assumption 2. The nonlinear function $g\left(x_{i}\right)$ satisfies the Lipschitz condition, i.e., there exists a constant $\sigma_{i}$ such that $0 \leq \frac{g_{i}\left(\varepsilon_{1}\right)-g_{i}\left(\varepsilon_{2}\right)}{\varepsilon_{1}-\varepsilon_{2}} \leq \sigma_{i}, \quad \forall \varepsilon_{1}, \varepsilon_{2} \in R, \varepsilon_{1} \neq \varepsilon_{2}, i=1,2, \ldots, N$.

To end this section, we introduce some lemmas for the proof of the main results in the paper.

Lemma 1 (see [8]). Let $b_{i}>0(i=1,2, \ldots, n)$ and $0<m<n$, then

$$
\left(\sum_{i=1}^{p} b_{i}^{n}\right)^{1 / n} \leq\left(\sum_{i=1}^{p} b_{i}^{m}\right)^{1 / m} .
$$

Lemma 2 (see [44]). Let $H, E$, and $F$ be real matrices of appropriate dimensions such that $F^{T} F \leq I$. Then, the following inequalities holds:

For any scalar $\gamma>0$ and vectors $X_{1}, X_{2} \in R^{n}$, $2 X_{1}^{T} H F E X_{2} \leq \gamma X_{1}^{T} H H^{T} X_{1}+\gamma^{-1} X_{2}^{T} E^{T} E X_{2}$.

Lemma 3 (see [45]). Assume that a continuous, positive definite function $V(t)$ satisfies the following differential inequality:

$$
\dot{V}(t) \leq-\omega V^{\rho}(t), \quad \forall t \geq t_{0}, V\left(t_{0}\right) \geq 0,
$$

where $\omega>0,0<\rho<1$ are constants. Then, for any given $t_{0}$, $V(t)$ satisfies the following inequality:

$$
\begin{cases}V^{1-\rho}(t) \leq V^{1-\rho}\left(t_{0}\right)-\omega(1-\rho)\left(t-t_{0}\right), & t_{0} \leq t \leq t^{*}, \\ V(t)=0, & t \geq t^{*},\end{cases}
$$

with $t^{*}$ given by $t^{*}=t_{0}+\left(V^{1-\rho}\left(t_{0}\right) / \omega(1-\rho)\right)$.

Lemma 4 (see [46]). For any $n$-dimension vector $X$ and vector $Y$, the following inequality is satisfied:

$$
2 X^{T} Y \leq \frac{1}{\gamma} X^{T} X+\gamma Y^{T} Y,
$$

where $\gamma$ is a suitable positive constant.

Lemma 5 (see [47]). Suppose there is a Lyapunov function $V(x)$ defined on a neighborhood $v \in \mathfrak{R}^{n}$ of the origin, and

$$
\dot{V}(x) \leq-\alpha V^{\eta}(x)+\theta V(x), \quad \forall x \in v \backslash\{0\} .
$$

Then, the origin of (1) is finite-time stable. The set

$$
\Omega=\left\{x \mid V(x)^{1-\eta}<\frac{\alpha}{\theta}\right\} \cap v,
$$

is contained in the domain of attraction of the origin. The settling time satisfies $T_{s} \leq\left(\left(\ln \left(1-(\theta / \alpha) V^{1-\eta}\left(x_{0}\right)\right)\right) / \theta\right.$ $(\eta-1)), x_{0} \in \Omega$.

\section{Main Result}

In this section, several sufficient conditions of finite-time synchronization for the uncertain complex network system under the controller (5) are provided by establishing a Lyapunov function. In the following analysis process, the time required for the system to achieve finite-time synchronization under different conditions or the upper bound of time required to be satisfied will be calculated.

Theorem 1. Under Assumptions 1 and 2, there exists a positive definite symmetric matrix $W$, so that the following inequality holds:

$$
\begin{aligned}
{\left[\begin{array}{cc}
I_{N} \otimes \Pi-2 W \otimes I_{n} & C \otimes I_{n} \\
* & 0
\end{array}\right] \leq } & 0, \\
\Pi= & A+A^{T}+H H^{T}+E_{1}^{T} E_{1}+L B \\
& +L\left(H H^{T}+E_{2}^{T} E_{2}\right)+L B^{T}, \\
W= & \operatorname{diag}\left\{w_{1}, w_{2}, \ldots, w_{N}\right\},
\end{aligned}
$$

then the controlled complex dynamic network (1) is synchronized with the given smooth dynamic $s(t)$ in a finite-time $t^{*}$ with

$$
t^{*}=\frac{V^{1-\delta}(0)}{2 k(1-\delta)},
$$

where $V(0)=\sum_{i=1}^{N} e_{i}^{T}(0) e_{i}(0)$ and $\delta=(1+\beta) / 2$.

Proof. Let $e(t)=\left(e_{1}^{T}(t), e_{2}^{T}(t), \ldots, e_{N}^{T}(t)\right)^{T}$, and construct the following Lyapunov function:

$$
V(t)=\sum_{i=1}^{N} e_{i}^{T}(t) e_{i}(t) .
$$

Calculating the time derivative of $V(t)$ along the trajectory of the error system (4) leads to 


$$
\begin{aligned}
\dot{V}(t)= & \sum_{i=1}^{N}\left[\dot{e}_{i}^{T}(t) e_{i}(t)+e_{i}^{T}(t) \dot{e}_{i}(t)\right] \\
= & \sum_{i=1}^{N}\left[(A+\Delta A(t)) e_{i}(t)+(B+\Delta B(t))\left(f\left(x_{i}(t)\right)-f(s(t))+\sum_{j=1}^{N} c_{i j} \phi\left(e_{j}\right)(t)-w_{i} e_{i}(t)-k \operatorname{sign}\left(e_{i}(t)\right)\left|e_{i}(t)\right|^{\beta}\right]^{T} e_{i}(t)\right. \\
& +\sum_{i=1}^{N} e_{i}^{T}(t)\left[(A+\Delta A(t)) e_{i}(t)+(B+\Delta B(t))\left(f\left(x_{i}(t)\right)-f(s(t))\right)+\sum_{j=1}^{N} c_{i j} \phi\left(e_{j}\right)(t)-w_{i} e_{i}(t)-k \operatorname{sign}\left(e_{i}(t)\right)\left|e_{i}(t)\right|^{\beta}\right] \\
= & \sum_{i=1}^{N}\left[e_{i}^{T}(t)\left[(A+\Delta A(t))^{T}+(A+\Delta A(t))-2 w_{i} I_{n}\right] e_{i}(t)+\left(f\left(x_{i}(t)\right)-f(s(t))\right)^{T}(B+\Delta B(t))^{T} e_{i}(t)\right. \\
& \left.+e_{i}^{T}(t)(B+\Delta B(t))\left(f\left(x_{i}(t)\right)-f(s(t))\right)\right]+\sum_{i=1}^{N} \sum_{j=1}^{N} \phi^{T}\left(e_{j}\right)(t) c_{i j}^{T} e_{i}(t)+\sum_{i=1}^{N} \sum_{j=1}^{N} e_{i}^{T}(t) c_{i j} \phi\left(e_{j}\right)(t) \\
& -\sum_{i=1}^{N} k \operatorname{sign}^{T}\left(e_{i}(t)\right)\left|e_{i}(t)\right|^{\beta} e_{i}(t)-\sum_{i=1}^{N} k e_{i}^{T}(t) \operatorname{sign}\left(e_{i}(t)\right)\left|e_{i}(t)\right|^{\beta} .
\end{aligned}
$$

From Assumption 1 and Lemma 2, we get

$$
\begin{aligned}
& e_{i}^{T}(t) \Delta A(t) e_{i}(t)=e_{i}^{T}(t) H F(t) E_{1} e_{i}(t) \leq \frac{1}{2}\left(e_{i}^{T}(t) H H^{T} e_{i}(t)+e_{i}^{T}(t) E_{1}^{T} E_{1} e_{i}(t)\right), \\
& e_{i}^{T}(t) \Delta A^{T}(t) e_{i}(t)=e_{i}^{T}(t) E_{1}^{T} F^{T}(t) H^{T} e_{i}(t) \leq \frac{1}{2}\left(e_{i}^{T}(t) E_{1}^{T} E_{1} e_{i}(t)+e_{i}^{T}(t) H H^{T} e_{i}(t)\right), \\
& e_{i}^{T}(t)\left[(A+\Delta A(t))^{T}+(A+\Delta A(t))\right] e_{i} \leq e_{i}^{T}(t)\left[A+A^{T}+H H^{T}+E_{1}^{T} E_{1}\right] e_{i}(t),
\end{aligned}
$$

Similarily, we can get that

$$
\begin{aligned}
( & \left.\left(x_{i}(t)\right)-f(s(t))\right)^{T}(B+\Delta B(t))^{T} e_{i}(t)+e_{i}^{T}(t)(B+\Delta B(t))\left(f\left(x_{i}(t)\right)-f(s(t))\right) \\
& \leq L e_{i}^{T}(t)\left[B+B^{T}+H H^{T}+E_{2}^{T} E_{2}\right] e_{i}(t), \\
\dot{V}(t) \leq & \sum_{i=1}^{N} e_{i}^{T}(t)\left[A+H H^{T}+E_{1}^{T} E_{1}+A^{T}+L B+L\left(H H^{T}+E_{2}^{T} E_{2}\right)+L B^{T}\right] e_{i}(t)+\sum_{i=1}^{N} \sum_{j=1}^{N} \phi^{T}\left(e_{j}\right)(t) c_{i j}^{T} e_{i}(t) \\
& +\sum_{i=1}^{N} \sum_{j=1}^{N} e_{i}^{T}(t) c_{i j} \phi\left(e_{j}\right)(t)-2 \sum_{i=1}^{N} e_{i}^{T}(t) w_{i} e_{i}(t)-\sum_{i=1}^{N} k \operatorname{sign}^{T}\left(e_{i}(t)\right)\left|e_{i}(t)\right|^{\beta} e_{i}(t)-\sum_{i=1}^{N} k e_{i}^{T}(t) \operatorname{sign}\left(e_{i}(t)\right)\left|e_{i}(t)\right|^{\beta} \\
= & \sum_{i=1}^{N} e_{i}^{T}(t)\left[A+H H^{T}+E_{1}^{T} E_{1}+A^{T}+L B+L\left(H H^{T}+E_{2}^{T} E_{2}\right)+L B^{T}\right] e_{i}(t)-2 \sum_{i=1}^{N} e_{i}^{T}(t) w_{i} e_{i}(t) \\
& +\sum_{i=1}^{N} \sum_{j=1}^{N} \phi^{T}\left(e_{j}\right)(t) c_{i j}^{T} e_{i}(t)+\sum_{i=1}^{N} \sum_{j=1}^{N} e_{i}^{T}(t) c_{i j} \phi\left(e_{j}\right)(t)-2 \sum_{i=1}^{N} k\left|e_{i}(t)\right|^{1+\beta} \\
= & \zeta^{T}(t) \Omega \tilde{\zeta}(t)-2 k \sum_{i=1}^{N}\left|e_{i}(t)\right|^{\beta+1},
\end{aligned}
$$


where $I$ is identity matrix with appropriate dimensions.

$$
\begin{aligned}
\zeta(t) & =\left[\begin{array}{ll}
e^{T}(t) & \phi^{T}(e(t))
\end{array}\right]^{T}, \widetilde{\zeta}(t)=[e(t) \phi(e(t))]^{T}, \\
\Omega & =\left(\begin{array}{cc}
I_{N} \otimes \Pi-2 W \otimes I_{n} & C \otimes I_{n} \\
* & 0
\end{array}\right), \\
\Pi & =A+H H^{T}+E_{1}^{T} E_{1}+A^{T}+L B+L\left(H H^{T}+E_{2}^{T} E_{2}\right)+L B^{T}, \\
W & =\operatorname{diag}\left(w_{1}, w_{2}, \ldots, w_{N}\right) .
\end{aligned}
$$

If the inequality (15) holds, then

$$
\dot{V}(t) \leq \zeta^{T}(t) \Omega \widetilde{\zeta}(t)-2 k \sum_{i=1}^{N} \sum_{j=1}^{N}\left|e_{i j}(t)\right|^{\beta+1} \leq-2 k \sum_{i=1}^{N} \sum_{j=1}^{N}\left|e_{i j}(t)\right|^{\beta+1} .
$$

Using Lemma 1, one has

$$
\begin{aligned}
\left(\sum_{i=1}^{N} \sum_{j=1}^{N}\left|e_{i j}(t)\right|^{\beta+1}\right)^{1 /(1+\beta)} & \geq\left(\sum_{i=1}^{N} \sum_{j=1}^{N}\left|e_{i j}(t)\right|^{2}\right)^{1 / 2}, \\
\sum_{i=1}^{N} \sum_{j=1}^{N}\left|e_{i j}(t)\right|^{\beta+1} & \geq\left(\sum_{i=1}^{N} \sum_{j=1}^{N}\left|e_{i j}(t)\right|\right)^{(1+\beta) / 2}=\left(\sum_{i=1}^{N} e_{i}^{T}(t) e_{i}(t)\right)^{(1+\beta) / 2} .
\end{aligned}
$$

In conclusion, we have

$$
\begin{aligned}
\dot{V}(t) & \leq-2 k \sum_{i=1}^{N} \sum_{j=1}^{N}\left|e_{i j}(t)\right|^{\beta+1} \leq-2 k\left(\sum_{i=1}^{N} e_{i}^{T}(t) e_{i}(t)\right)^{(1+\beta) / 2} \\
& =-2 k V^{(1+\beta) / 2}(t) .
\end{aligned}
$$

From Lemma 3, $V(t)$ converges to zero in a finite time $t^{*}$, where $t^{*}$ is given by

$$
t^{*}=\frac{V^{1-\delta}(0)}{2 k(1-\delta)}
$$

where $V(0)=\sum_{i=1}^{N} e_{i}^{T}(0) e_{i}(0)$ and $\delta=(1+\beta) / 2$.

Remark 3. The results of the finite-time synchronization of the complex dynamic network model studied are abundant, but the results of the finite-time synchronization of complex network models with uncertain parameters are scant $[17,33,38-41]$. In $[17,33,40]$, infinite-time synchronization was studied with consideration of the incoherent matrix of complex network models being uncertain. In [30, 41], the finite-time synchronization of uncertain complex network systems was investigated. In [30], the dynamic behavior function of a single node containing unknown parameters was considered, and the internal coupling matrix was linearly uncertain. In [41], switching control was studied, and in both of the abovementioned references, eigenvalue characterization was used. Our models and research methods are different.

Corollary 1. If Assumptions 1 and 2 are established and there exist a positive definite symmetric matrix $W$ such that

$$
\lambda_{\max }(M)-\lambda_{\min }(2 W) \leq 0
$$

where

$$
\begin{aligned}
M= & I_{N} \otimes\left(A+H H^{T}+E_{1}^{T} E_{1}+A^{T}+L B+L\left(H H^{T}+E_{2}^{T} E_{2}\right)+L B^{T}\right) \\
& +\left((C \Sigma)^{T}(C \Sigma)+I_{N}\right) \otimes I_{n}, \\
\Sigma= & \operatorname{diag}\left(\sigma_{1}, \sigma_{2}, \ldots, \sigma_{N}\right), W=\operatorname{diag}\left(w_{1}, w_{2}, \ldots, w_{N}\right),
\end{aligned}
$$

where $\lambda_{\max }(M)$ is the maximum eigenvalue of the matrix $M$ and $\lambda_{\min }(W)$ is the minimum eigenvalue of the matrix $W$, then the system (1) can achieve finite-time synchronization under the controller $u_{i}(t)$. The synchronization time $t^{*}$ is obtained as

$$
t^{*}=\frac{V^{1-\delta}(0)}{2 k(1-\delta)}
$$

where $V(0)=\sum_{i=1}^{N} e_{i}^{T}(0) e_{i}(0)$ and $\delta=(1+\beta) / 2$.

Proof. The proof is similar to Theorem 2 and it is omitted.

If the uncertainties of the complex dynamic networks are not considered, the complex dynamic network model is given as follows:

$$
\begin{aligned}
\dot{x}_{i}(t) & =A x_{i}(t)+B f\left(x_{i}(t)\right)+\sum_{j=1}^{N} c_{i j} g\left(x_{j}(t)\right)+u_{i}(t), \\
i & =1,2, \ldots, N .
\end{aligned}
$$

In this situation, the error system becomes

$$
\dot{e}_{i}(t)=A e_{i}(t)+B\left(f\left(x_{i}(t)\right)-f(s(t))\right)+\sum_{j=1}^{N} c_{i j} \phi\left(e_{j}\right)(t)+u_{i}(t) .
$$

For the complex network model (29), we can obtain the following result. 
Corollary 2. Under Assumptions 1 and 2, if there exists a positive definite symmetric matrix $W$, so that the inequality (15) is satisfied, where $\Pi=A+A^{T}+L B+L B^{T}$, then we can conclude that the system (29) can achieve finite-time synchronization under the controller $u_{i}(t)$. The settling time is obtained as

$$
t^{*}=\frac{V^{1-\delta}(0)}{2 k(1-\delta)},
$$

where $V(0)=\sum_{i=1}^{N} e_{i}^{T}(0) e_{i}(0)$ and $\delta=(1+\beta) / 2$.

Remark 4. At present, the research results of complex dynamic networks without uncertainties are abundant. However, these results are different from those of previous research. For example, the state variable of the ith node was not considered in [25], and only the linear coupling relationship between nodes was recognized. A study [?] used nonperiodic intermittent control to enable a complex network system to reach finite-time synchronization; however, each node in the system under consideration was in the same state. In [48], the complex network model and research method are different from that in the present study.

If $\Gamma$ represents the internal coupling between the state variables of each node, the complex dynamic network model is as follows:

$$
\begin{aligned}
\dot{x}_{i}(t)= & (A+\Delta A(t)) x_{i}(t)+(B+\Delta B(t)) f\left(x_{i}(t)\right) \\
& +c \sum_{j=1}^{N} d_{i j} \Gamma x_{j}(t)+u_{i}(t),
\end{aligned}
$$

where the real number $c$ is the coupling strength; $\Gamma \in R^{n \times n}$ is the inner connecting matrix between nodes; the constant matrix $D=\left(d_{i j}\right)_{N \times N}$ represents the system's linear coupling matrix, and the definition of $d_{i j}$ is as follows: if a connection exists between node $i$ and node $j(i \neq j)$, then $d_{i j}>0$; otherwise, $d_{i j}=0$. The diagonal elements of matrix $D$ are defined as follows: $d_{i i}=-\sum_{j=1, j \neq i}^{N} d_{i j}$. Then, the error system reads

$$
\begin{aligned}
\dot{e}_{i}(t)= & (A+\Delta A(t)) e_{i}(t)+(B+\Delta B(t))\left(f\left(x_{i}(t)\right)-f(s(t))\right) \\
& +c \sum_{j=1}^{N} d_{i j} \Gamma e_{j}(t)+u_{i}(t) .
\end{aligned}
$$

For the complex dynamic model (32), we can make the following results.

Corollary 3. Suppose that Assumptions 1 and 2 hold, and a positive definite symmetric matrix $W$ exists such that the following inequality is satisfied:

$$
\begin{gathered}
I_{N} \otimes\left[A+A^{T}+H H^{T}+E_{1}^{T} E_{1}+L B+L\left(H H^{T}+E_{2}^{T} E_{2}\right)+L B^{T}\right] \\
-2 W \otimes I_{n}+c\left(D \otimes \Gamma+D^{T} \otimes \Gamma^{T}\right) \leq 0,
\end{gathered}
$$

then system (32) can reach the finite-time synchronization state under the controller (5), and the settling time is obtained as follows:

$$
t^{*}=\frac{V^{1-\delta}(0)}{2 k(1-\delta)},
$$

where $V(0)=\sum_{i=1}^{N} e_{i}^{T}(0) e_{i}(0)$ and $\delta=(1+\beta) / 2$.

Remark 5. Existing studies on the finite-time synchronization of complex dynamic networks considered the coupling relationship between nodes as a linear one [28-30, 32, 34, 41, 49]. While regarding the uncertainty and dynamic behavior of a system, the results for a prefunction matrix as a nonidentity matrix are few. In $[25,30,32]$, the uncertainty of a system and the dynamic matrix of the dynamic behavior function were not considered as the identity matrix. A neural network model was used in [34].

If the uncertainties of complex dynamic networks are not considered, the error system (33) can be rewritten as follows:

$$
\begin{aligned}
\dot{e}_{i}(t)= & A e_{i}(t)+B\left(f\left(x_{i}(t)\right)-f(s(t))\right) \\
& +c \sum_{j=1}^{N} d_{i j} \Gamma e_{j}(t)+u_{i}(t) .
\end{aligned}
$$

Corollary 4. Assume that Assumptions 1 and 2 hold, and the following inequality is satisfied:

$$
\begin{aligned}
I_{N} & \otimes\left(A+A^{T}+L B+L B^{T}\right)-2 W \otimes I_{n} \\
& +c\left(D \otimes \Gamma+D^{T} \otimes \Gamma^{T}\right) \leq 0
\end{aligned}
$$

then the error system (36) can achieve finite-time synchronization under the controller $u_{i}(t)$. Also, the settling time is as follows:

$$
t^{*}=\frac{V^{1-\delta}(0)}{2 k(1-\delta)},
$$

where $V(0)=\sum_{i=1}^{N} e_{i}^{T}(0) e_{i}(0)$ and $\delta=(1+\beta) / 2$.

Theorem 2. Under Assumptions 1 and 2, if there exists a positive definite symmetric matrix $W$ such that

$$
\lambda_{\max }(M)-\lambda_{\min }(2 W)>0,
$$

where

$$
\begin{aligned}
M= & I_{N} \otimes\left[A+H H^{T}+E_{1}^{T} E_{1}+A^{T}+H H^{T}+E_{1}^{T} E_{1}+L B\right. \\
& \left.+L\left(H H^{T}+E_{2}^{T} E_{2}\right)+L B^{T}\right]+\left[(C \Sigma)^{T}(C \Sigma)+I_{N}\right] \otimes I_{n} \\
\Sigma= & \operatorname{diag}\left(\sigma_{1}, \sigma_{2}, \ldots, \sigma_{N}\right), W=\operatorname{diag}\left(w_{1}, w_{2}, \ldots, w_{N}\right),
\end{aligned}
$$


with $I_{N}$ being the $N$-dimension identity matrix, then the controlled complex dynamic network (1) is synchronized with the given smooth dynamic $s(t)$ in a finite time less than $T_{s}$ as follows:

$$
T_{\mathrm{s}} \leq \frac{2 \ln \left(1-(1 / 2 k)\left(\lambda_{\max }(M)-\lambda_{\min }(2 W)\right) V^{(1-\beta) / 2}(0)\right)}{\left(\lambda_{\max }(M)-\lambda_{\min }(2 W)\right)(\beta-1)},
$$

Proof. Let $e(t)=\left(e_{1}^{T}(t), e_{2}^{T}(t), \ldots, e_{N}^{T}(t)\right)^{T}$, and construct the following Lyapunov function:

$$
V(t)=\sum_{i=1}^{N} e_{i}^{T}(t) e_{i}(t)
$$

Calculating the time derivative of $V(t)$ along the trajectory of the error system (4) leads to

where $V(0)=(1 / 2) \sum_{i=1}^{N} e_{i}^{T}(0) e_{i}(0)$.

$$
\begin{aligned}
\dot{V}(t)= & \sum_{i=1}^{N}\left[\dot{e}_{i}^{T}(t) e_{i}(t)+e_{i}^{T}(t) \dot{e}_{i}(t)\right] \\
= & \sum_{i=1}^{N}\left[(A+\Delta A(t)) e_{i}(t)+(B+\Delta B(t))\left(f\left(x_{i}(t)\right)-f(s(t))\right)+\sum_{j=1}^{N} c_{i j} \phi\left(e_{j}\right)(t)-w_{i} e_{i}(t)-k \operatorname{sign}\left(e_{i}(t)\right)\left|e_{i}(t)\right|^{\beta}\right]^{T} e_{i}(t) \\
& +\sum_{i=1}^{N} e_{i}^{T}(t)\left[(A+\Delta A(t)) e_{i}(t)+(B+\Delta B(t))\left(f\left(x_{i}(t)\right)-f(s(t))\right)+\sum_{j=1}^{N} c_{i j} \phi\left(e_{j}\right)(t)-w_{i} e_{i}(t)-k \operatorname{sign}\left(e_{i}(t)\right)\left|e_{i}(t)\right|^{\beta}\right] .
\end{aligned}
$$

By Assumption 2 and Lemma 4, one gets

$$
\begin{gathered}
\phi\left(e_{i}(t)\right)=g\left(x_{i}(t)\right)-g(s(t)) \leq \sigma_{i} e_{i}(t), \\
\phi^{T}(e(t))\left(C^{T} \otimes I_{n}\right) e(t)+e^{T}(t)\left(C \otimes I_{n}\right) \phi(e(t)) \leq \frac{1}{2}\left[\phi^{T}(e(t))\left(\left(C^{T} C\right) \otimes I_{n}\right) \phi(e(t))+e^{T}(t) e(t)\right] \\
+\frac{1}{2}\left[\phi^{T}(e(t))\left(\left(C^{T} C\right) \otimes I_{n}\right) \phi(e(t))+e^{T}(t) e(t)\right] \\
\leq \phi^{T}(e(t))\left(\left(C^{T} C\right) \otimes I_{n}\right) \phi(e(t))+e^{T}(t) e(t) \leq e^{T}(t)\left(\left[(C \Sigma)^{T}(C \Sigma)+I_{N}\right] \otimes I_{n}\right) e(t) .
\end{gathered}
$$

Thus,

$$
\begin{aligned}
\dot{V}(t) \leq & \sum_{i=1}^{N} e_{i}^{T}(t)\left[A+H H^{T}+E_{1}^{T} E_{1}+A^{T}+L B+L\left(H H^{T}+E_{2}^{T} E_{2}\right)+L B^{T}-2 w_{i} I_{n}\right] e_{i}(t)+\sum_{i=1}^{N} \sum_{j=1}^{N} c_{i j}^{T} \phi^{T}\left(e_{j}\right)(t) e_{i}(t) \\
& +\sum_{i=1}^{N} \sum_{j=1}^{N} e_{i}^{T}(t) c_{i j} \phi\left(e_{j}\right)(t)-\sum_{i=1}^{N} k \operatorname{sign}^{T}\left(e_{i}(t)\right)\left|e_{i}(t)\right|^{\beta} e_{i}(t)-\sum_{i=1}^{N} k e_{i}^{T}(t) \operatorname{sign}\left(e_{i}(t)\right)\left|e_{i}(t)\right|^{\beta} \\
= & e^{T}(t)\left[I_{N} \otimes\left[A+H H^{T}+E_{1}^{T} E_{1}+A^{T}+L B+L\left(H H^{T}+E_{2}^{T} E_{2}\right)+L B^{T}\right]+\left[(C \Sigma)^{T}(C \Sigma)+I_{N}\right] \otimes I_{n}-2 W \otimes I_{n}\right] e(t) \\
& -2 k \sum_{i=1}^{N}\left|e_{i}(t)\right|^{1+\beta} \\
= & e^{T}(t)\left(M-2 W \otimes I_{n}\right) e(t)-2 k \sum_{i=1}^{N}\left|e_{i}(t)\right|^{1+\beta}
\end{aligned}
$$


where $C$ is a symmetric matrix, and

$$
\begin{aligned}
M & =I_{N} \otimes\left[A+H H^{T}+E_{1}^{T} E_{1}+A^{T}+H H^{T}+E_{1}^{T} E_{1}+L B+L\left(H H^{T}+E_{2}^{T} E_{2}\right)+L B^{T}\right]+\left[(C \Sigma)^{T}(C \Sigma)+I_{N}\right] \otimes I_{n}, \\
\Sigma & =\operatorname{diag}\left\{\sigma_{1}, \sigma_{2}, \ldots, \sigma_{N}\right\}, W=\operatorname{diag}\left\{w_{1}, w_{2}, \ldots, w_{N}\right\} .
\end{aligned}
$$

According to the inequality (39), one has

$$
\begin{aligned}
\dot{V}(t) & \leq e^{T}(t)\left(\lambda_{\max }(M)-\lambda_{\min }(2 W)\right) e(t)-2 k \sum_{i=1}^{N} \sum_{j=1}^{N}\left|e_{i j}(t)\right|^{\beta+1} \\
& =\sum_{i=1}^{N} e_{i}^{T}(t)\left(\lambda_{\max }(M)-\lambda_{\min }(2 W)\right) e_{i}(t)-2 k \sum_{i=1}^{N} \sum_{j=1}^{N}\left|e_{i j}(t)\right|^{\beta+1} .
\end{aligned}
$$

From (23), we can get that

$$
\begin{aligned}
\dot{V}(t) & \leq\left(\lambda_{\max }(M)-\lambda_{\min }(2 W)\right) V(t)-2 k V^{(1+\beta) / 2}(t) \\
& =-\alpha(V(t))^{(1+\beta) / 2}+\theta V(t),
\end{aligned}
$$

where $\alpha=2 k$ and $\theta=\left(\lambda_{\max }(M)-\lambda_{\min }(2 W)\right)$.

According to Lemma 5, the system (1) can reach the state of synchronization in finite time $t^{*} \leq T_{s}$ as

$$
T_{s} \leq \frac{2 \ln \left(1-(1 / 2 k)\left(\lambda_{\max }(M)-\lambda_{\min }(2 W)\right) V^{(1-\beta) / 2}(0)\right)}{\left(\lambda_{\max }(M)-\lambda_{\min }(2 W)\right)(\beta-1)},
$$

where $V(0)=\sum_{i=1}^{N} e_{i}^{T}(0) e_{i}(0)$.

Remark 6. In the proof of Corollary 1, under the condition that the inequality (26) is assumed to be established, considering the influence of $M-2 W$ on the system is not necessary. The sufficient conditions for the system to achieve finite-time synchronization are obtained. In the proof of Theorem 2, we consider the influence of matrix $M-2 W$ on the conditions that the system must satisfy to achieve finite-time synchronization and reduce conservativeness.

Remark 7. In the proof result of Theorem 1, we obtain the exact time required for the uncertain complex dynamic network (1) to achieve the finite-time synchronization. In Theorem 2, we find the upper bound of the time required for the system to reach the synchronization state under other conditions. For some cases where the exact synchronization time needs to be known, the conclusion of Theorem 1 is more in line with the requirements. For others where there is no strict requirement for synchronization time or the eigenvalue of matrix is easier to be obtained, the conclusion of Theorem 2 has stronger applicability.

If the uncertainties of time-varying complex dynamic networks are not considered, the complex dynamic network model is shown in (29). According to the network model, we can obtain the following conclusion.

Corollary 5. Under Assumptions 1 and 2, if a positive definite symmetric matrix $W$ exists and results in inequality
(39), where $M=I_{N} \otimes\left[A+A^{T}+L B+L B^{T}\right]+\left[(C \Sigma)^{T}(C \Sigma)+\right.$ $\left.I_{N}\right] \otimes I_{n}$, then the system (29) can reach finite-time synchronization under the controller (5). The finite-time $T_{s}$ is as follows:

$$
T_{\mathrm{s}} \leq \frac{2 \ln \left(1-(1 / 2 k)\left(\lambda_{\max }(M)-\lambda_{\min }(2 W)\right) V^{(1-\beta) / 2}(0)\right)}{\left(\lambda_{\max }(M)-\lambda_{\min }(2 W)\right)(\beta-1)} .
$$

When the coupling relationship between state variables of each node in the complex network system is linear, the state equation and error equation of the system are shown in (32) and (33). For this system, we can draw the following conclusions.

Corollary 6. Under Assumptions 1 and 2, assume that there exists a positive definite symmetric matrix $W$ such that

$$
\lambda_{\Theta}+\epsilon \lambda_{D+D^{T}}-2 \omega>0
$$

where $\Theta=A+A^{T}+H H^{T}+E_{1}^{T} E_{1}+L B+L\left(H H^{T}+E_{2}^{T}\right.$ $\left.E_{2}\right)+L B^{T}, \varepsilon=c\|\Gamma\|_{2}, \lambda_{\Theta}=\lambda_{\max }(\Theta), \lambda_{D+D^{T}}=\lambda_{\max }\left(D+D^{T}\right)$, and $\omega=\min \left\{w_{i}\right\}$. Then, under the control of $u_{i}$, the system (32) can reach the finite-time synchronization state, and the settling time $t^{*} \leq T_{s}$ is obtained as

$$
T_{s} \leq \frac{2 \ln \left(1-(1 / 2 k)\left(\lambda_{\Theta}+\epsilon \lambda_{D+D^{T}}-2 \omega\right) V^{(1-\beta) / 2}(0)\right)}{\left(\lambda_{\Theta}+\epsilon \lambda_{D+D^{T}}-2 \omega\right)(\beta-1)} .
$$

If the uncertainties of time-varying complex dynamic networks are not considered and the internal coupling between the state variables of each node is linear, the error state equation of the complex network system is shown as in (36). In this time, the following can concluded:

Corollary 7. Under Assumptions 1 and 2, if there exists a positive definite symmetric matrix $W$ satisfying the inequality

$$
\lambda_{\Lambda}+\epsilon \lambda_{D+D^{T}}-2 \omega>0
$$

where $\Lambda=A+A^{T}+L B+L B^{T}$ and $\varepsilon=c\|\Gamma\|_{2}$, then the system (36) can achieve the finite-time synchronization. Also, the settling time is obtained as follows:

$$
T_{\mathrm{s}} \leq \frac{2 \ln \left(1-(1 / 2 k)\left(\lambda_{\Lambda}+\epsilon \lambda_{D+D^{T}}-2 \omega\right) V^{(1-\beta) / 2}(0)\right)}{\left(\lambda_{\Lambda}+\epsilon \lambda_{D+D^{T}}-2 \omega\right)(\beta-1)},
$$




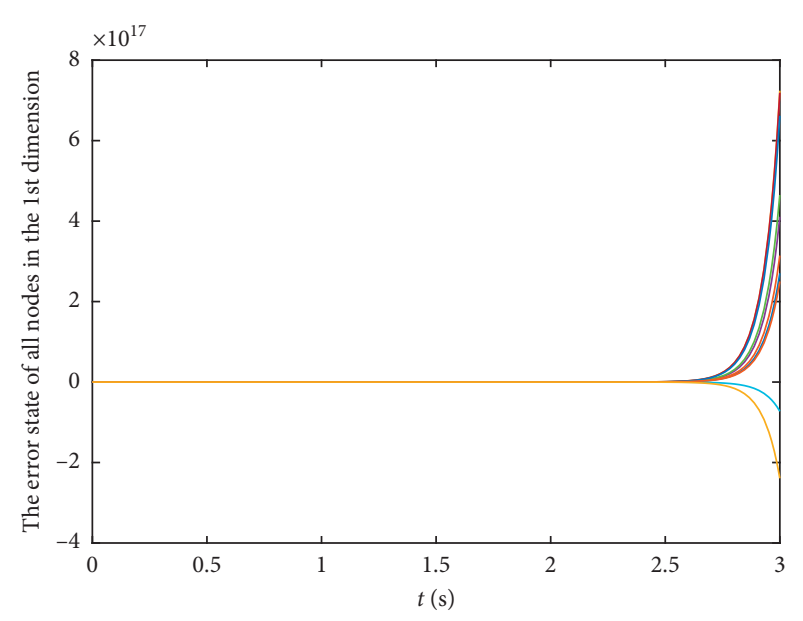

(a)

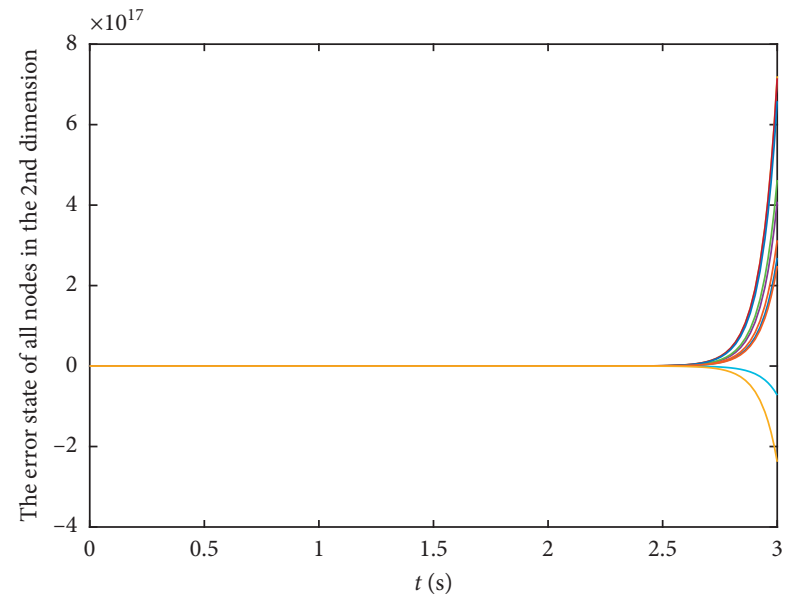

(b)

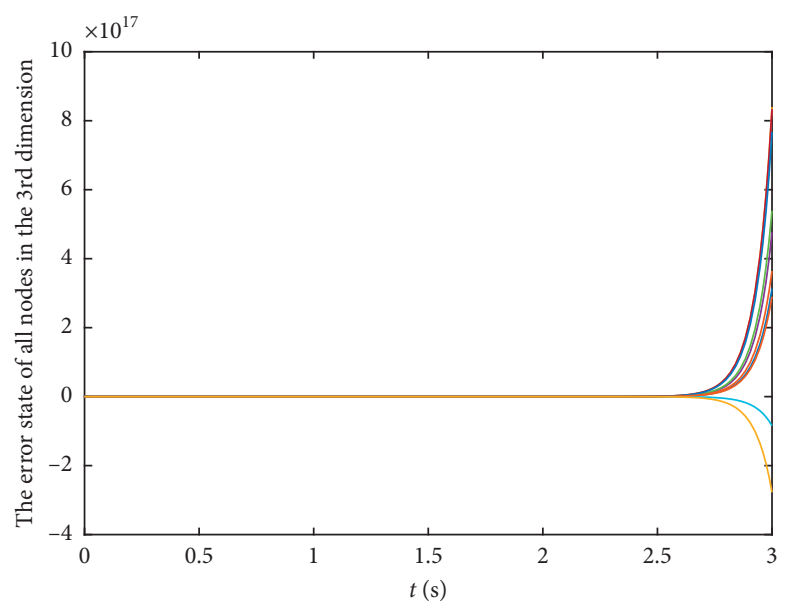

(c)

FIgURE 1: The state of all nodes in different dimensions without the controller.

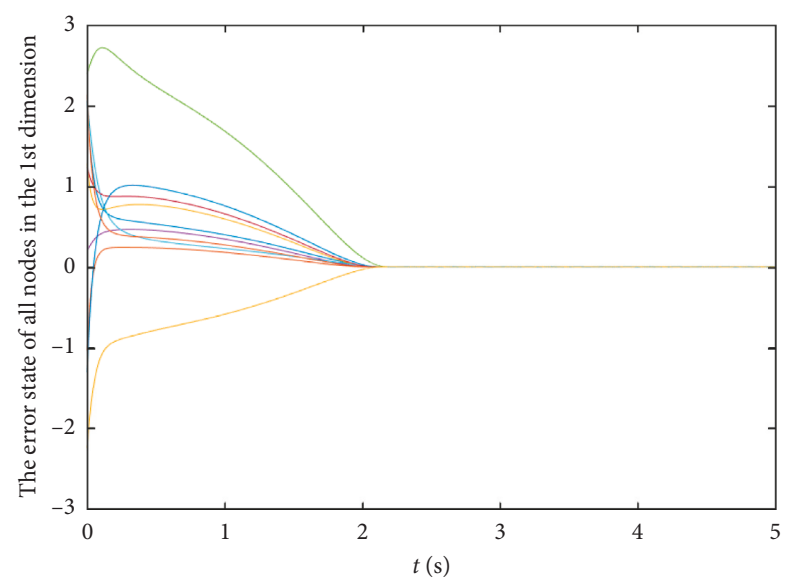

(a)

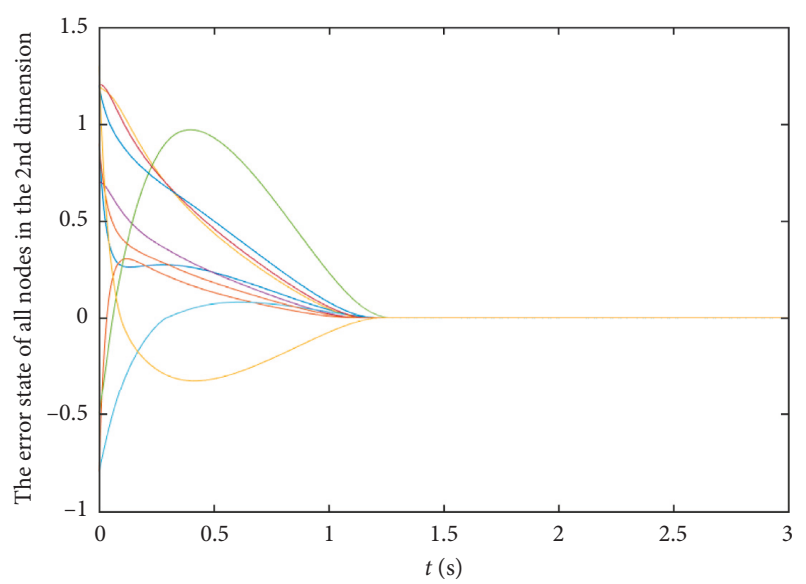

(b)

Figure 2: Continued. 


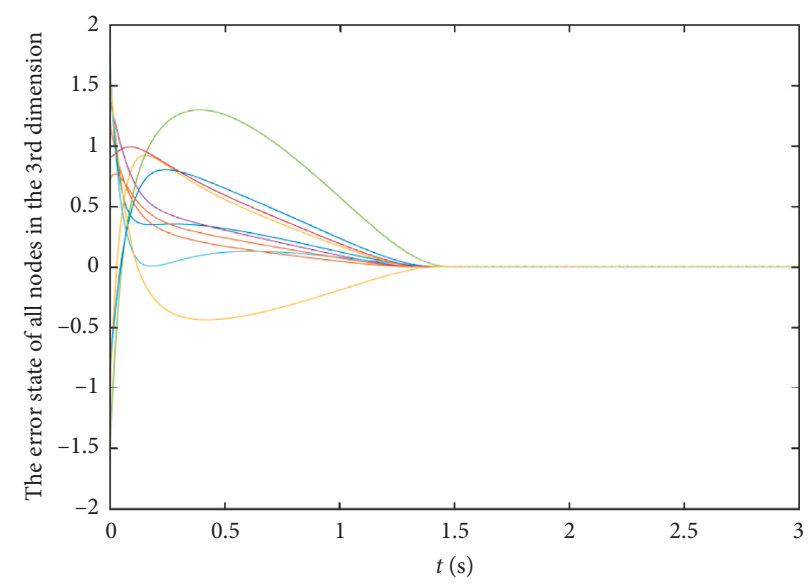

(c)

Figure 2: The error state of all nodes in different dimensions under the controller (5).

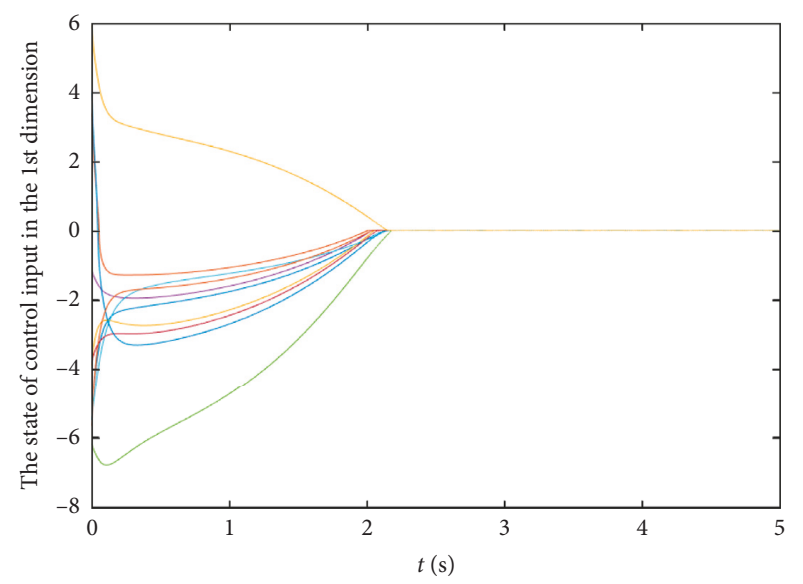

(a)

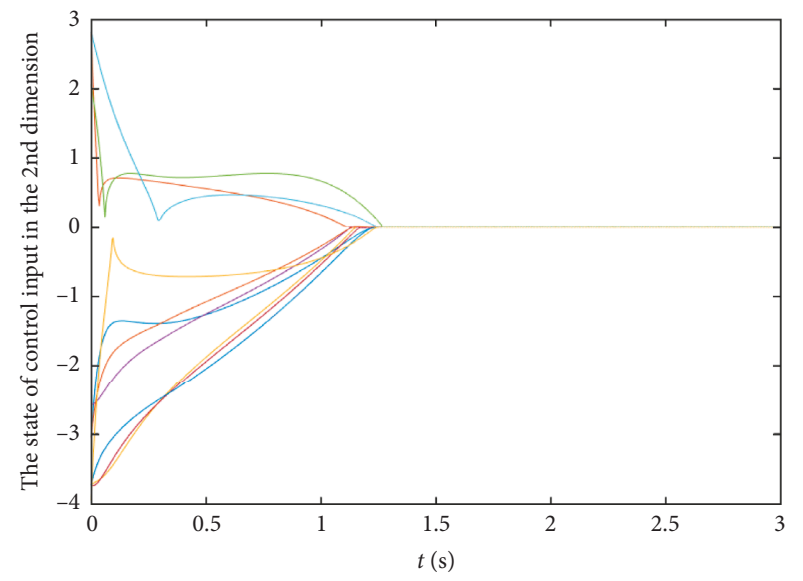

(b)

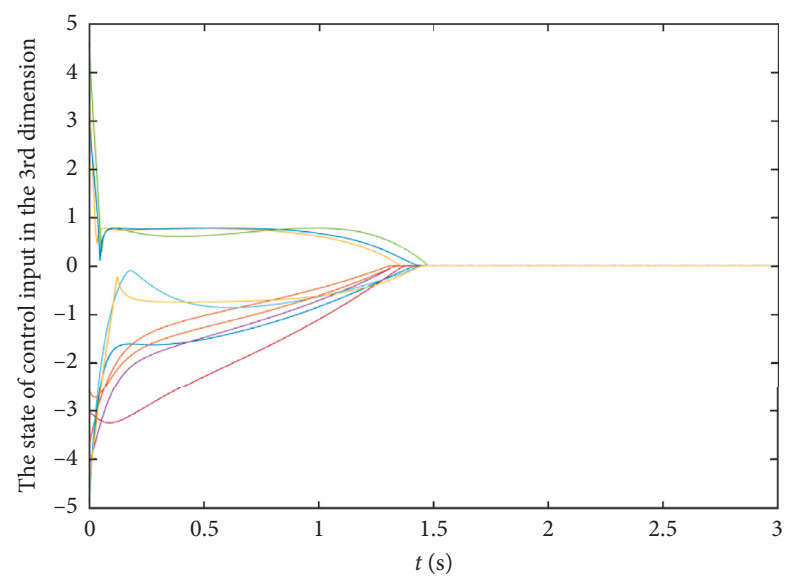

(c)

Figure 3: The state of the input $u_{i}$ in different dimensions. 
where $V(0)=\sum_{i=1}^{N} e_{i}^{T}(0) e_{i}(0)$.

Corollary 8. Assume that $\lambda_{\max }(M-2 W)>0$, where $W=\operatorname{diag}\left(w_{1}, w_{2}, \ldots, w_{N}\right)$. If an appropriate matrix $W$ exists and satisfies this inequality, then the system (1) can realize synchronization in finite time. The time used to realize synchronization is as follows:

$$
T_{s} \leq \frac{2 \ln \left(1-(1 / 2 k)\left(\lambda_{\max }(M-2 W)\right) V^{(1-\beta) / 2}(0)\right)}{\left(\lambda_{\max }(M-2 W)\right)(\beta-1)},
$$

where $V(0)=\sum_{i=1}^{N} e_{i}^{T}(0) e_{i}(0)$.

Remark 8. In the previous theorem and the corollary verification process, the eigenvalues of $M$ are the maximum values, and the eigenvalues of $W$ are the minimum values. In the present case, the eigenvalues of $M$ and $W$ are the maximum.

\section{Numerical Simulation}

In this section, we verify the theoretical results by numerical simulation.

Suppose that a network model consists of 10 nodes, the internal dimension of each node is 3 , the coupling relationship between the nodes is nonlinear, and the system contains uncertainties. The state equation of each node is described as follows:

$$
\begin{aligned}
\dot{x}_{i}(t)= & (A+\Delta A(t)) x_{i}(t)+(B+\Delta B(t)) f\left(x_{i}(t)\right) \\
& +\sum_{j=1}^{10} c_{i j} g\left(x_{j}(t)\right)+u_{i}(t), \quad i=1,2, \ldots, 10,
\end{aligned}
$$

where $A, B, C, H, E_{1}, E_{2}$, and $\Sigma$ are the parameter values in the system given as

$$
\begin{aligned}
& A=\left[\begin{array}{ccc}
-3 & 4 & 1 \\
4 & -2 & 2 \\
1 & 2 & -2
\end{array}\right], \\
& B=\left[\begin{array}{ccc}
-5 & 1 & -1 \\
1 & -2 & 1 \\
-1 & 1 & -3
\end{array}\right], \\
& H=\left[\begin{array}{ccc}
-0.1 & 0 & 0 \\
0 & -1 & 1 \\
1 & 0 & -1
\end{array}\right], \\
& E_{1}=\left[\begin{array}{ccc}
-4 & 5 & 3 \\
0 & 1 & 0 \\
1 & 0 & 4
\end{array}\right], \\
& E_{2}=\left[\begin{array}{ccc}
1 & 0 & 2 \\
-1 & -1 & 2 \\
1 & 0 & 1
\end{array}\right],
\end{aligned}
$$

$$
C=\left[\begin{array}{cccccccccc}
-3 & 1 & 1 & 0 & -1 & 1 & 2 & 0 & -3 & 2 \\
1 & -2 & 1 & 1 & 2 & -1 & -3 & 0 & -1 & 2 \\
1 & 2 & -5 & 2 & 0 & -1 & -3 & 0 & 2 & 2 \\
0 & 1 & -2 & 0 & -2 & 2 & -1 & -1 & 0 & 3 \\
-1 & 2 & 1 & -2 & -1 & 1 & 0 & 0 & -1 & 1 \\
1 & -1 & -1 & 2 & 1 & -2 & 0 & -3 & 2 & 1 \\
2 & -3 & -1 & -1 & 0 & 0 & 2 & 1 & 2 & -2 \\
1 & 1 & -3 & 1 & 3 & -3 & 1 & -2 & -1 & 2 \\
-3 & -1 & 1 & 3 & -3 & 2 & -2 & 1 & 3 & -1 \\
0 & 2 & -2 & 1 & 1 & 0 & 1 & 2 & 0 & -5
\end{array}\right]
$$

$$
\begin{aligned}
\Sigma & =\operatorname{diag}([1,1.5,0.7,0.8,1,1.2,0.9,0.8,1.1,1.4]), \\
I_{N} & =\operatorname{diag}([1,1,1,1,1,1,1,1,1,1]), \\
I_{n} & =\operatorname{diag}([1,1,1]), \\
F(t) & =\operatorname{diag}([\sin (t), \sin (2 t), \sin (3 t)]) .
\end{aligned}
$$

The nonlinear vector-valued function in the complex network is described by $f(x)=\tanh (x), g(x)=\sin (x)$. The matrix $W$ and the controller gain $k$ that satisfy the conditions in Theorem 1 can be found using the MATLAB toolbox. The motion trajectory of the error system can be obtained, as shown in the figures. Figure 1 shows the error state curve of all nodes in different dimensions without the controller, while Figure 2 represents the error state under the action of the controller (5). Meanwhile, Figure 3 shows the state of the control input $u_{i}$ of all nodes. From these figures, it is clear that under the obtained controllers, each node of the system can achieve synchronization within the finite time.

According to the initial value data, when $k=2$ and $\beta=0.4$, the time required for the first, second, and third dimension of all nodes to reach the synchronization state is $t_{1}=2.157, t_{2}=1.209$, and $t_{3}=1.506$, respectively. Meanwhile, when MATLAB was used for simulation, the synchronization time corresponding to that mentioned above is $t_{1}^{*}=2.133, t_{2}^{*}=1.244$, and $t_{3}^{*}=1.445$, respectively. By comparing the synchronization time of the two cases, it can be seen that the estimated value is basically consistent with the simulation value. Therefore, the feasibility of the theoretical method proposed in this paper has been verified.

\section{Conclusion}

This study investigates the finite-time synchronization control problem for a class of nonlinear uncertain complex dynamic networks. In the complex network model, the uncertainty of the system is considered, and the appropriate Lyapunov function is constructed under the assumption that the uncertain term satisfies the matching condition. An appropriate feedback control is designed on the basis of the linear matrix inequality and Lipschitz inequality. Some sufficient criterions are obtained for guaranteeing finite-time synchronization of a class of complex dynamic networks with parameters uncertainties under different conditions. According to this, an estimation of the upper bound of the 
time required to synchronize the system is given. Finally, the feasibility and effectiveness of the proposed method are verified by numerical simulation.

In the upcoming research, we will focus on the following aspects:

(1) The uncertainties considered in this paper are to satisfy both the norm bounded and matching conditions. In the following work, the case where the uncertain term only satisfies the norm bounded or only knows the upper or lower bounds of the system parameters will be studied.

(2) The method of using the strategy of pinning control to realize finite-time synchronization of uncertain complex dynamic networks will be taken into account.

(3) Event-triggered finite-time synchronization control for uncertain complex networks will be studied.

\section{Data Availability}

The data used to support the findings of this study are available from the corresponding author upon request.

\section{Conflicts of Interest}

The authors declare that there are no conflicts of interest regarding the publication of this paper.

\section{Authors' Contributions}

Both authors contributed equally to the writing of this paper. Furthermore, both authors also read carefully and approved the final manuscript.

\section{Acknowledgments}

This work was supported by the National Natural Science Foundation of China (grant no. 11372107), the National Science Foundation of China (grant no. 11972156), and the Natural Science Foundation of Hunan Province (grant no. 2017JJ4004).

\section{References}

[1] X.-M. Zhang, Q.-L. Han, and J. Wang, "Admissible delay upper bounds for global asymptotic stability of neural networks with time-varying delays," IEEE Transactions on Neural Networks and Learning Systems, vol. 29, no. 11, pp. 5319-5329, 2018.

[2] L. Ding, Q.-L. Han, and X.-M. Zhang, "Distributed secondary control for active power sharing and frequency regulation in islanded microgrids using an event-triggered communication mechanism," IEEE Transactions on Industrial Informatics, vol. 15, no. 7, pp. 3910-3922, 2018.

[3] J. Lehnert, "Synchronization in complex networks," in Controlling Synchronization Patterns in Complex Networks, pp. 33-41, Springer International Publishing, New York, NY, USA, 2016.

[4] C. Bernadette and M. Shlomo, "Synchronization in dynamic networks," 2017, https://arxiv.org/abs/1706.07831.
[5] X.-M. Zhang, Q.-L. Han, Z. Wang, and B.-L. Zhang, "Neuronal state estimation for neural networks with two additive time-varying delay components," IEEE Transactions on Cybernetics, vol. 47, no. 10, pp. 3184-3194, 2017.

[6] X.-M. Zhang and Q.-L. Han, "Global asymptotic stability analysis for delayed neural networks using a matrix-based quadratic convex approach," Neural Networks, vol. 54, pp. 57-69, 2014.

[7] G.-R. Chen, "Problems and challenges in control theory under complex dynamical network environments," Acta Automatica Sinica, vol. 39, no. 4, pp. 312-321, 2013.

[8] G. Cai, H. Zhao, and L. Zhang, "Adaptive cluster synchronization of uncertain complex dynamic networks in finite time," International Journal of Nonlinear Science, vol. 21, no. 3, pp. 181-186, 2016.

[9] T. Liu, G. M. Dimirovski, and J. Zhao, "Exponential synchronization of complex delayed dynamic networks with general topology," Physica Section A: Statistical and Theoretical Physics, vol. 387, no. 2-3, pp. 643-652, 2008.

[10] X. Wu and H. Lu, "Exponential synchronization of weighted general delay coupled and non-delay coupled dynamical networks," Computers \& Mathematics with Applications, vol. 60, no. 8, pp. 2476-2487, 2010.

[11] H.-H. Lian, S.-P. Xiao, Z. Wang, X.-H. Zhang, and H.-Q. Xiao, "Further results on sampled-data synchronization control for chaotic neural networks with actuator saturation," Neurocomputing, vol. 346, pp. 30-37, 2019.

[12] L. Zhou, J. She, S. Zhou, and C. Li, "Compensation for statedependent nonlinearity in a modified repetitive control system," International Journal of Robust and Nonlinear Control, vol. 28, no. 1, pp. 213-226, 2018.

[13] B. Kaviarasan, R. Sakthivel, and Y. Lim, "Synchronization of complex dynamical networks with uncertain inner coupling and successive delays based on passivity theory," Neurocomputing, vol. 186, pp. 127-138, 2016.

[14] L. Zhou, L. Cheng, J. She, and Z. Zhang, "Generalized extended state observer-based repetitive control for systems with mismatched disturbances," International Journal of Robust and Nonlinear Control, vol. 29, no. 11, pp. 3777-3792, 2019.

[15] B.-L. Zhang, Q.-L. Han, X.-M. Zhang, and X. Yu, "Sliding mode control with mixed current and delayed states for offshore steel jacket platforms," IEEE Transactions on Control Systems Technology, vol. 22, no. 5, pp. 1769-1783, 2014.

[16] J. Wang, H. Zhang, Z. Wang, and B. Wang, "Local exponential synchronization in complex dynamical networks with timevarying delay and hybrid coupling," Applied Mathematics and Computation, vol. 225, pp. 16-32, 2013.

[17] R. Rakkiyappan, G. Velmurugan, J. Nicholas George, and R. Selvamani, "Exponential synchronization of Lur'e complex dynamical networks with uncertain inner coupling and pinning impulsive control," Applied Mathematics and Computation, vol. 307, pp. 217-231, 2017.

[18] H. Zhao and G. Cai, "Exponential synchronization of complex delayed dynamical networks with uncertain parameters via intermittent control," Advances in Neural Networks C ISNN 2015, Springer International Publishing, New York, NY, USA, pp. 91-98, 2015.

[19] Y. Wu and L. Liu, "Exponential outer synchronization between two uncertain time-varying complex networks with nonlinear coupling," Entropy, vol. 17, no. 5, pp. 3097-3109, 2015.

[20] J. Lu, J. Chen, and W. Chen, "Global asymptotical synchronization of delayed complex dynamical networks with 
switching topology," IFAC Proceedings Volumes, vol. 46, no. 13, pp. 206-211, 2013.

[21] M. Syed Ali, L. Palanisamy, J. Yogambigai, and L. Wang, "Passivity-based synchronization of Markovian jump complex dynamical networks with time-varying delays, parameter uncertainties, reaction-diffusion terms, and sampled-data control," Journal of Computational and Applied Mathematics, vol. 352, pp. 79-92, 2019.

[22] H. Zhao, L. Li, H. Peng et al., "Finite-time synchronization for multi-link complex networks via discontinuous control," Optik, vol. 138, pp. 440-454, 2017.

[23] W. Zhang, C. Li, X. He, and H. Li, "Finite-time synchronization of complex networks with non-identical nodes and impulsive disturbance," Modern Physics Letter B, vol. 32, no. 1, Article ID 1850002, 2018.

[24] W. Zhang, C. Li, T. Huang, and J. Huang, "Fixed-time synchronization of complex networks with nonidentical nodes and stochastic noise perturbations," Physica A: Statistical Mechanics and Its Applications, vol. 492, pp. 15311542, 2018.

[25] A. Muhammadhaji, A. Abdurahman, and H. Jiang, "Finitetime synchronization of complex dynamical networks with time-varying delays and nonidentical nodes," Journal of Control Science and Engineering, vol. 2017, Article ID 5072308, 13 pages, 2017.

[26] J. Sun, Y. Wang, Y. Wang, and Y. Shen, "Finite-time synchronization between two complex-variable chaotic systems with unknown parameters via nonsingular terminal sliding mode control," Nonlinear Dynamics, vol. 85, no. 2, pp. 1105-1117, 2016.

[27] S. Sheng, X. Zhang, and G. Lu, "Finite-time outer-synchronization for complex networks with Markov jump topology via hybrid control and its application to image encryption," Journal of the Franklin Institute, vol. 355, no. 14, pp. 64936519, 2018.

[28] F. Xiao, Q.-T. Gan, and Q. Yuan, "Finite-time cluster synchronization for time-varying delayed complex dynamical networks via hybrid control," Advances in Difference Equations, vol. 2019, no. 1, p. 93, 2019.

[29] N. Gunasekaran, R. Saravanakumar, Y. H. Joo, and H. S. Kim, "Finite-time synchronization of sampled-data T-S fuzzy complex dynamical networks subject to average dwell-time approach," Fuzzy Sets and Systems, vol. 374, pp. 40-59, 2019.

[30] M. Zhang and M. Han, "Finite-time synchronization of uncertain complex networks with nonidentical nodes based on a special unilateral coupling control," in Proceedings of the 2017 International Symposium on Neural Networks, Springer, Hokkaido, Japan, June 2017.

[31] J. Mei, M. Jiang, and J. Wang, "Finite-time structure identification and synchronization of drive-response systems with uncertain parameter," Communications in Nonlinear Science and Numerical Simulation, vol. 18, no. 4, pp. 999-1015, 2013.

[32] R. Xu, Y. Kao, and M. Gao, "Finite-time synchronization of Markovian jump complex networks with generally uncertain transition rates," Transactions of the Institute of Measurement and Control, vol. 39, no. 1, pp. 52-60, 2017.

[33] N. Ma, Z. Liu, and L. Chen, "Robust and non-fragile finite time $H_{\infty}$ synchronization control for complex networks with uncertain inner coupling," Computational and Applied Mathematics, vol. 37, no. 4, pp. 5395-5409, 2018.

[34] H. Zhao, M. Zheng, S. Li, and W. Wang, "New results on finite-time parameter identification and synchronization of uncertain complex dynamical networks with perturbation,"
Modern Physics Letters B, vol. 32, no. 9, Article ID 1850112, 2018.

[35] J. Sun, Y. Wu, G. Cui, and Y. Wang, "Finite-time real combination synchronization of three complex-variable chaotic systems with unknown parameters via sliding mode control," Nonlinear Dynamics, vol. 88, no. 3, pp. 1677-1690, 2017.

[36] J. Mei, M. Jiang, W. Xu, and B. Wang, "Finite-time synchronization control of complex dynamical networks with time delay," Communications in Nonlinear Science and $\mathrm{Nu}$ merical Simulation, vol. 18, no. 9, pp. 2462-2478, 2013.

[37] Q. Li, J. Guo, C. Sun, Y. Wu, and Z. Ding, "Finite-time synchronization for a class of dynamical complex networks with nonidentical nodes and uncertain disturbance," Journal of Systems Science and Complexity, vol. 32, no. 3, pp. 818-834, 2019.

[38] T. Guo, D. Cao, Y. Yang, S. Liu, and W. Huang, "Robust synchronization of impulsively coupled complex dynamical network with delayed nonidentical nodes," Chaos, Solitons and Fractals, vol. 87, pp. 92-101, 2016.

[39] C. Huang, D. W. C. Ho, and J. Lu, "Partial-information-based synchronization analysis for complex dynamical networks," Journal of the Franklin Institute, vol. 352, no. 9, pp. 34583475, 2015.

[40] Y.-P. Zhao, P. He, H. Saberi Nik, and J. Ren, "Robust adaptive synchronization of uncertain complex networks with multiple time-varying coupled delays," Complexity, vol. 20, no. 6, pp. 62-73, 2015.

[41] Y. Wu, J. Cao, Q. Li, A. Alsaedi, and F. E. Alsaadi, "Finite-time synchronization of uncertain coupled switched neural networks under asynchronous switching," Neural Networks, vol. 85, pp. 128-139, 2017.

[42] M. Syed Ali and J. Yogambigai, "Extended dissipative synchronization of complex dynamical networks with additive time-varying delay and discrete-time information," Journal of Computational and Applied Mathematics, vol. 348, pp. 328$341,2019$.

[43] Z. Cao, C. Li, X. Wang, and T. Huang, "Finite-time consensus of linear multi-agent system via distributed event-triggered strategy," Journal of the Franklin Institute, vol. 355, no. 3, pp. 1338-1350, 2018.

[44] Y. Wang, L. Xie, and C. E. de Souza, "Robust control of a class of uncertain nonlinear systems," Systems \& Control Letters, vol. 19, no. 2, pp. 139-149, 1992.

[45] Y. Tang, "Terminal sliding mode control for rigid robots," Automatica, vol. 34, no. 1, pp. 51-56, 1998.

[46] S. Liu and P. Liu, "Adaptive anti-synchronization of chaotic complex nonlinear systems with unknown parameters," Nonlinear Analysis: Real World Applications, vol. 12, no. 6, pp. 3046-3055, 2011.

[47] Y. Shen and X. Xia, "Semi-global finite-time observers for nonlinear systems," Automatica, vol. 44, no. 12, pp. 31523156, 2008.

[48] Z. Cai, X. Pan, L. Huang, and J. Huang, "Finite-time robust synchronization for discontinuous neural networks with mixed-delays and uncertain external perturbations," Neurocomputing, vol. 275, pp. 2624-2634, 2018.

[49] M. Zhang and M. Han, "Finite-time projective synchronization control of uncertain complex networks with brushless DC motor and Rikitake system," in Proceedings of IEEE 2016 8th International Conference on Advanced Computational Intelligence, pp. 100-107, IEEE, Chiang Mai, Thailand, February 2016. 


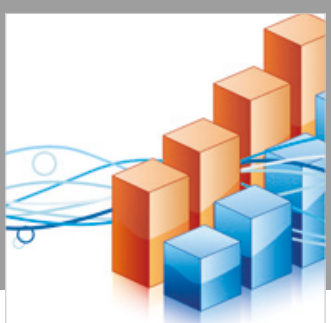

Advances in

Operations Research

\section{-n-m}
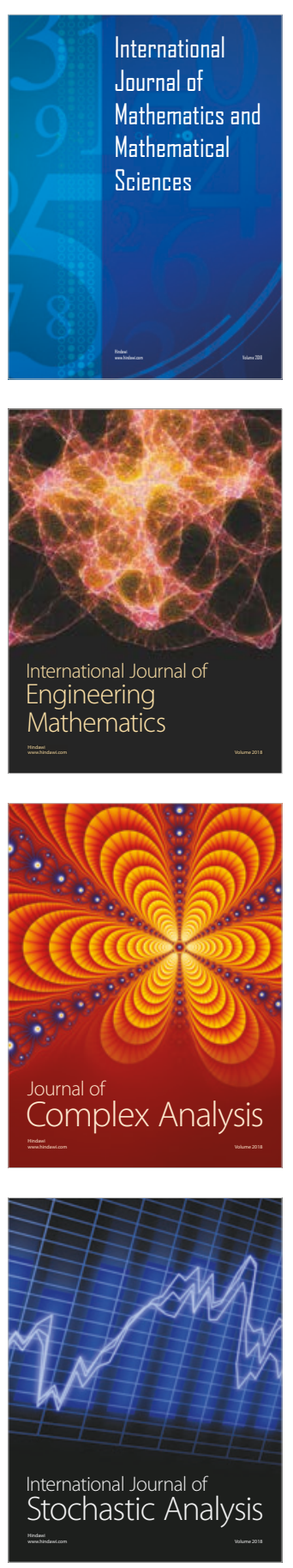
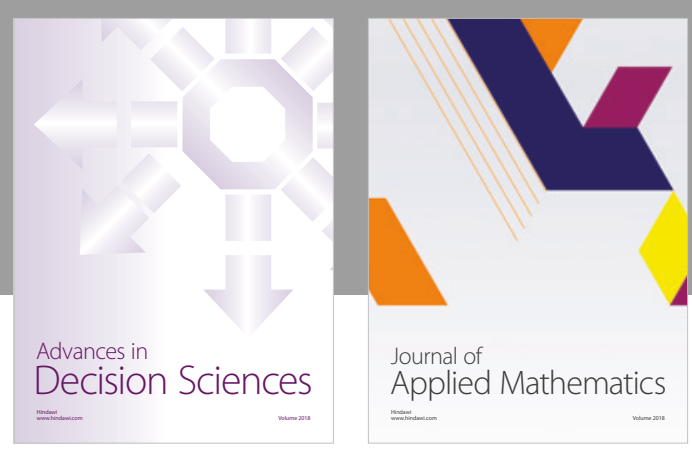

Journal of

Applied Mathematics
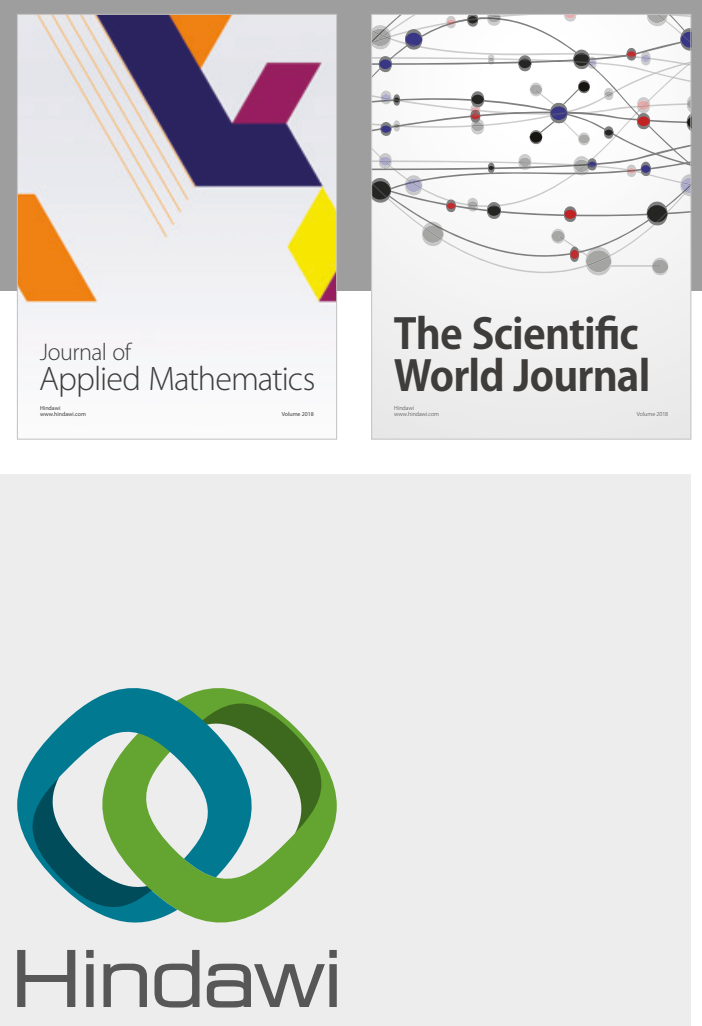

Submit your manuscripts at

www.hindawi.com

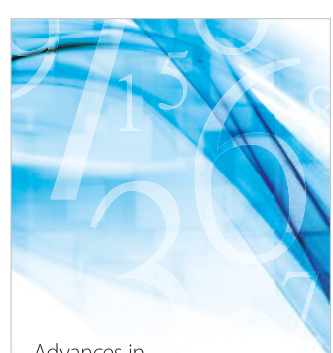

Advances in
Numerical Analysis
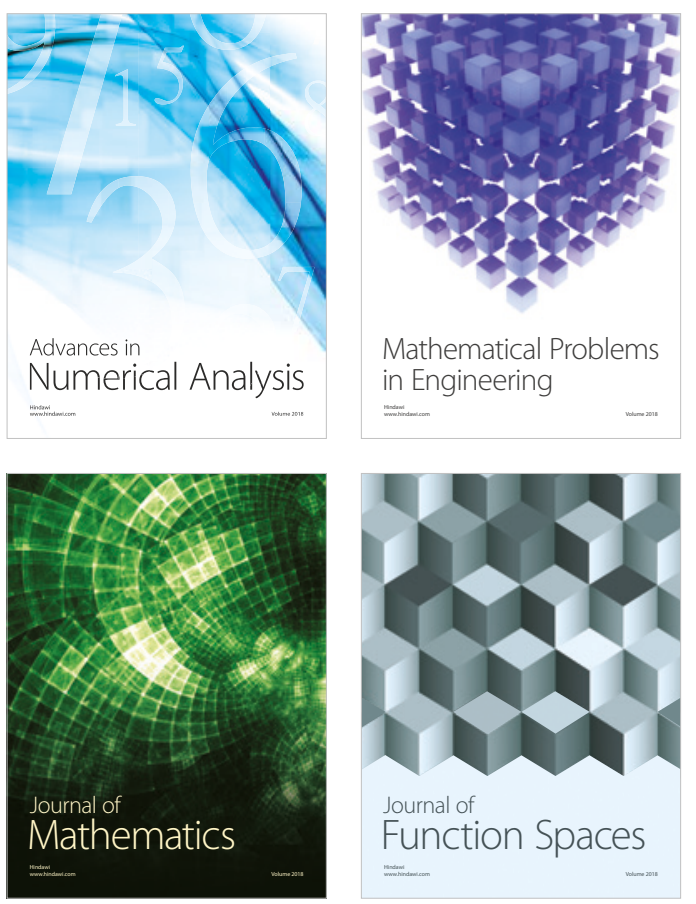

Mathematical Problems in Engineering

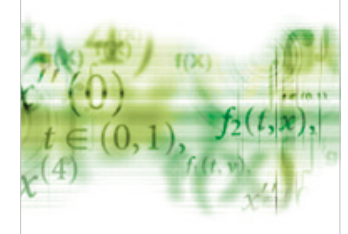

International Journal of

Differential Equations

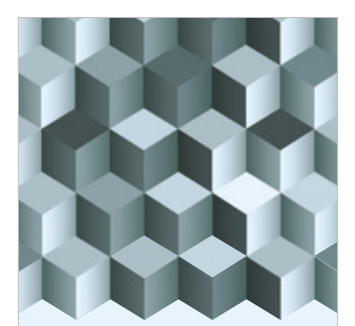

Journal of

Function Spaces
The Scientific

World Journal

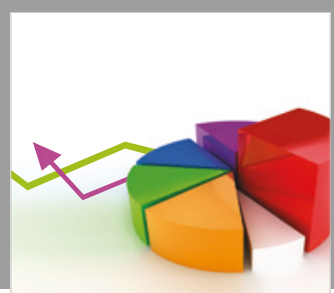

Journal of

Probability and Statistics
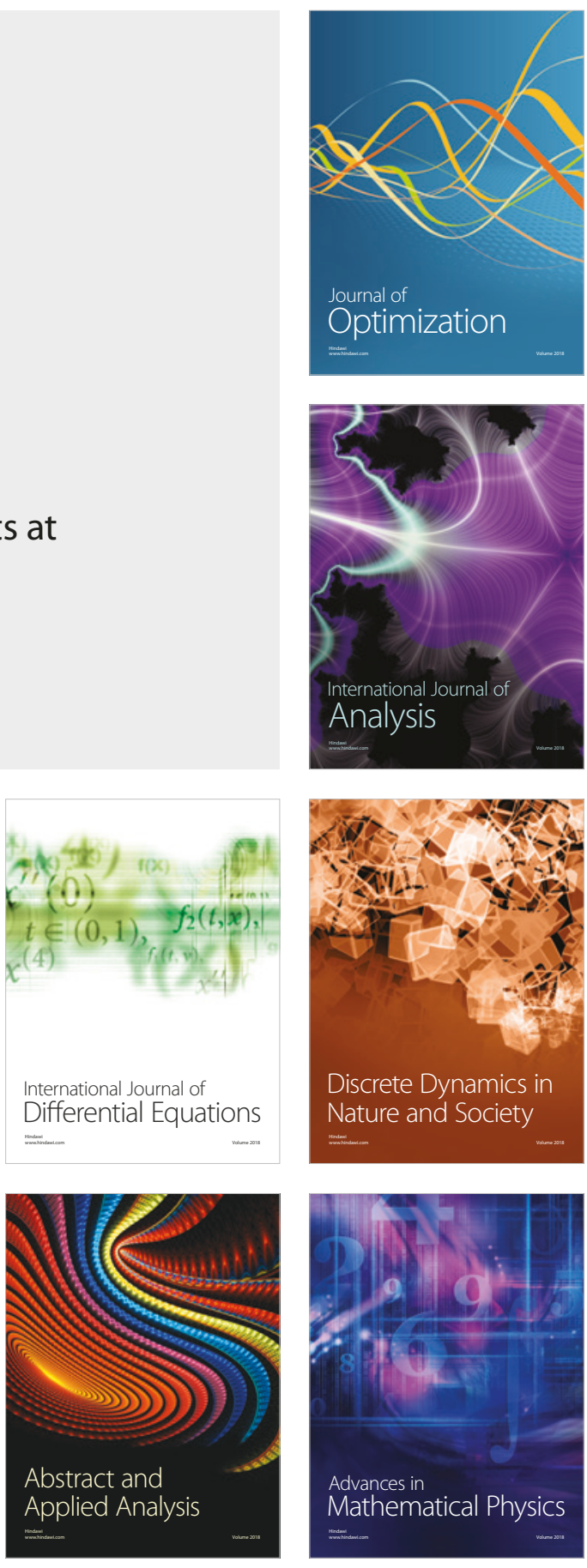